\title{
Her2/neu signaling blockade improves tumor oxygenation in a multifactorial fashion in $\mathrm{Her} 2 / \mathrm{neu}^{+}$tumors
}

\author{
Matthew E. Hardee $\cdot$ Rose J. Eapen · Zahid N. Rabbani • \\ Matthew R. Dreher · Jeffrey Marks · Kimberly L. Blackwell • \\ Mark W. Dewhirst
}

Received: 26 December 2007/ Accepted: 4 March 2008/Published online: 26 March 2008

(C) The Author(s) 2008

\begin{abstract}
Purpose Tumor hypoxia reduces the efficacy of radiation and chemotherapy as well as altering gene expression that promotes cell survival and metastasis. The growth factor receptor, Her2/neu, is overexpressed in $25-30 \%$ of breast tumors. Tumors that are Her $2^{+}$may have an altered state of oxygenation, relative to Her2 ${ }^{-}$tumors, due to differences in tumor growth rate and angiogenesis.

Methods Her2 blockade was accomplished using an antibody to the receptor (trastuzumab; Herceptin). This study examined the effects of Her2 blockade on tumor angiogenesis, vascular architecture, and hypoxia in Her2 ${ }^{+}$ and Her2 ${ }^{-}$MCF7 xenograft tumors.
\end{abstract}

\section{E. Hardee $\cdot$ M. W. Dewhirst}

Department of Pathology, Duke University Medical Center,

Durham, NC, USA

\section{R. J. Eapen}

Department of Otolaryngology, University of North Carolina Medical Center, Chapel Hill, NC, USA

Z. N. Rabbani · K. L. Blackwell · M. W. Dewhirst $(\bowtie)$ Department of Radiation Oncology, Duke University Medical Center, Durham, NC 22710, USA

e-mail: dewhi001@mc.duke.edu; dewhirst@ radonc.duke.edu

\section{R. Dreher}

Department of Diagnostic Radiology, National Institutes of Health Clinical Center, Bethesda, MD, USA

J. Marks

Department of Surgery, Duke University Medical Center, Durham, NC, USA

K. L. Blackwell

Department of Medicine, Duke University Medical Center, Durham, NC, USA
Results Treatment with trastuzumab in $\mathrm{Her}^{+}$tumors significantly improved tumor oxygenation, increased microvessel density, and improved vascular architecture compared with the control-treated $\mathrm{Her}^{+}$tumors. The $\mathrm{Her}^{+}$xenografts treated with trastuzumab also demonstrated decreased proliferation indices when compared with control-treated xenografts. These results indicate that Her2 blockade can improve tumor oxygenation by decreasing oxygen consumption (reducing tumor cell proliferation and inducing necrosis) and increasing oxygen delivery (vascular density and architecture).

Conclusions These results support the use of trastuzumab as an adjunct in the treatment of breast tumors with chemotherapy or radiotherapy, as improvements in tumor oxygenation should translate into improved treatment response.

Keywords Her2/neu - Angiogenesis - Tumor hypoxia . Breast cancer

\section{Introduction}

It is well established that tumor hypoxia is associated with treatment resistance, aggressive clinical phenotype, and poor prognosis $[1,2]$. There are multiple reasons for the negative effect of hypoxia on treatment response, including direct interference with the treatment efficacy of radiation and drugs, and altered gene expression that promotes angiogenesis, cell survival, and metastasis [3-8]. Hypoxiaresponsive genes are involved in many cell functions: cell cycle control, apoptosis regulation, angiogenesis, regulation of metabolism, and protection of cells from oxidative stress. The role of hypoxia in tumor multi-drug resistance, invasiveness, angiogenesis, and metastasis is currently an area of intense research interest. 
The factors that contribute most to limiting oxygen transport are low vascular density coupled with irregular geometry and oxygen consumption that is out of balance with supply $[9,10]$. Oxygen consumption rates in proliferating cells are 3-5 times greater than cells in $\mathrm{G}_{\mathrm{o}}[11,12]$, and variations in oxygen consumption rate of this order of magnitude would be expected to completely dominate oxygen transport, producing hypoxic tissue [9]. Low vascular density and increased $\mathrm{O}_{2}$ consumption are affected by Her2/neu expression in tumors. Laboratory studies have shown that Her 2 overexpression increases breast tumor cell proliferation which should increase oxygen consumption rates [13-16]. However, Her2 overexpression can also drive angiogenesis by increasing expression of the proangiogenic cytokine vascular endothelial growth factor (VEGF), independent of hypoxic upregulation [17]. Petit et al. reported the first observation of a relationship between Her2 overexpression and VEGF. In this report, the use of trastuzumab in Her2 positive cell lines reduced VEGF expression. Other studies have demonstrated that Her2 and EGFR overexpression can lead to increased levels of HIF-1 and VEGF [18, 19].

Because inhibition of Her2 signaling can inhibit both cell proliferation and angiogenesis, predictions about changes in tumor oxygenation following inhibition of Her2 signaling are difficult to make. In an attempt to determine the change in the net balance between oxygen delivery and consumption, this study examines the effect of Her2 blockade on intratumoral $\mathrm{pO}_{2}$, microvessel density, tumor vascular architecture, necrosis and tumor cell proliferation in a model of $\mathrm{Her}^{+}$and Her2 ${ }^{-}$human breast tumor xenografts. The reasons for an increase in tumor oxygenation following trastuzumab treatment are multifactorial. Improvements in vascular density and organization as well as reduction in oxygen consumption rate all contribute to the overall increase in tumor $\mathrm{pO}_{2}$.

\section{Materials and methods}

\section{Tumor model}

Two MCF7 human breast cancer cell lines were used in all experiments: parent $\mathrm{MCF}^{\mathrm{WT}}$ cell line (Her2 nonexpressing) and $\mathrm{MCF}^{\mathrm{Her} 2}$, which is a stable cell line that has been transfected to constitutively overexpress Her2 (Fig. 1).

The $\mathrm{MCF}^{\mathrm{Her} 2}$ line was created by transfection of pcDNA3.1 containing the full-length wild type coding sequence of human ERBB2. In parallel, cells were also transfected with the empty vector. After 2 days, cells were selected in RPMI containing 10\% FBS and $600 \mu \mathrm{g} / \mathrm{ml}$ G418 (Mediatech, Herndon, VA) until colonies of

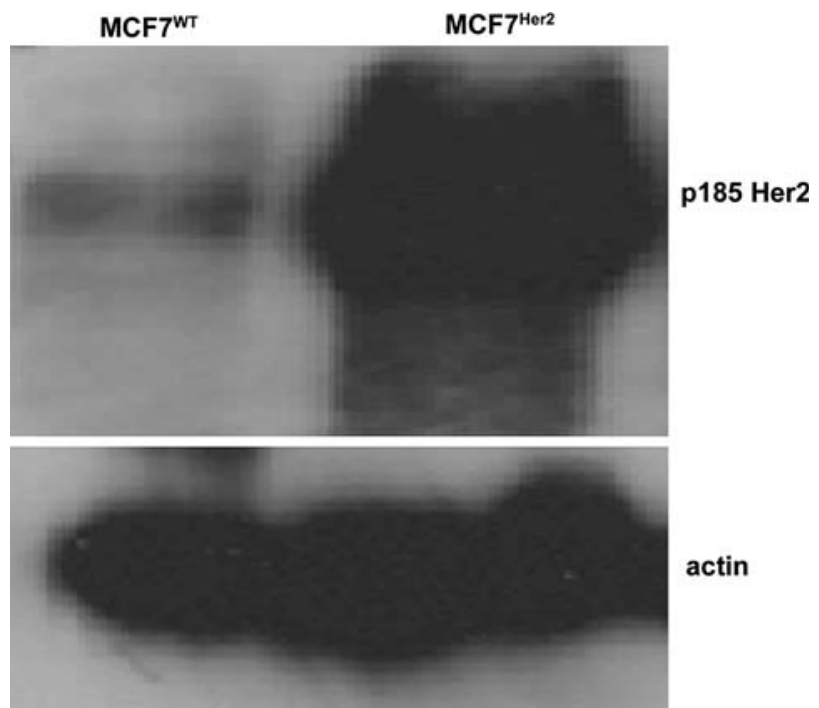

Fig. 1 Transfected MCF7-Her2 cells overexpress Her2. Shown is an immunoblot of $\mathrm{MCF} 7^{\mathrm{WT}}$ and transfected $\mathrm{MCF} 7^{\mathrm{Her} 2}$ tumor cells for p185/Her2, along with actin loading control

approximately 200 cells developed. Single colonies from both the ERBB2 and empty vector were isolated by cloning cylinders, transferred to 24 well dishes and grown in medium containing G418. These clones were then assayed for ERBB2 expression by immunoblotting and comparison to the parental line and empty vector clones. After selection, cells are maintained in medium containing $100 \mu \mathrm{g} / \mathrm{ml}$ G418. The clone selected for these studies expresses ERBB2 protein at approximately 100 -fold over the parental and empty vector control cells. Further, by FISH analysis, it was determined that these cells contain 13-14 copies of the ERBB2 coding sequence.

All animal experiments were performed in accordance with Duke University's institutional animal care and use committee. After estrogen pellet implantation (Innovative Research of America, Sarasota, FL), female nude mice (BALB/c nu/nu), weighing approximately $20 \mathrm{~g}$ were injected with $10^{7}$ tumor cells subcutaneously into the left flank. Tumors volumes were measured until they reached $100 \mathrm{~mm}^{3}$. Animals were then randomized to Her2 blockade (trastuzumab $10 \mathrm{mg} / \mathrm{kg}$ IP daily) or control (Human Myeloma $\operatorname{IgG}_{1} 10 \mathrm{mg} / \mathrm{kg}$ IP daily; Biodesign, Saco, ME). Treatment was continued for 2 weeks. Tumor dimensions were measured thrice per week, and volume was calculated by the following formula:

Volume $=$ length $\times$ width $\times$ height $\times \pi / 6$

Intratumoral $\mathrm{pO}_{2}$ measurements

Following the 2-week treatment with trastuzumab or placebo, animals were anesthetized with nembutal $75 \mathrm{mg} / \mathrm{kg}$ and placed on a heating pad. Oxygen measurements were 
performed using a fiber optic oxygen-sensing device (Oxford Optronix, Oxylite). Two profiles were obtained with a micromanipulator, using a $50 \mu \mathrm{m}$ step distance (200-300 measurements per tumor). Following $\mathrm{pO}_{2}$ measurements, the animals were euthanized with an overdose of nembutal.

\section{Tumor excision}

Additional animals were administered pimonidazole hydrochloride (Natural Pharmaceuticals, International Inc., Belmont, MA) $70 \mathrm{mg} / \mathrm{kg}$ intraperitoneally, for immunohistochemical determination of hypoxia. After 3-4 h, the mice were anesthetized with nembutal at $10 \mathrm{mg} / \mathrm{kg}$ and placed on a heating pad for tumor excision. After tumor excision, specimens were immediately snap-frozen. Complete tumor sections from 4 or 5 animals in each group (8-10 $\mu \mathrm{m}$ thickness) were cut and stored at $-80^{\circ} \mathrm{C}$ until further processing. Each of the tumors was stained and analyzed for hypoxia (pimonidazole), endothelial structures (CD31), proliferation (Ki67), and VEGF expression. Tumor sections were also stained with hematoxylin and eosin (H\&E) to distinguish viable from necrotic tissue.

\section{Immunohistochemistry}

Immunohistochemistry was performed using protocols designed for single images or whole tumor sections.

For single image analysis, endogenous peroxidase activity was quenched with $3 \%$ hydrogen peroxide for $15 \mathrm{~min}$, followed by blocking with $10 \%$ donkey serum for $15 \mathrm{~min}$. The slides were then incubated with primary antibodies: anti-rabbit VEGF antibody (Santa Cruz, dilution 1:400) overnight at $4^{\circ} \mathrm{C}$; anti-Ki67 rabbit monoclonal antibody (Vector Lab; dilution 1:4,000) overnight at $4^{\circ} \mathrm{C}$; and mouse monoclonal anti-pimonidazole (hypoxyprobe-1 (Chemicon; Temecula, CA); dilution 1:100). Washing was accomplished with phosphate-buffered saline (PBS). Omission of the primary antibody served as negative control. Secondary antibodies were applied for $1 \mathrm{~h}$ as follows: biotinylated donkey anti-rabbit antibody (VEGF, Ki67); Cy-3-conjugated donkey anti-mouse antibody (pimonidazole; 1:200 for $2 \mathrm{~h}$; Chemicon). Visualization of Ki67 and VEGF staining was accomplished with ABC kit (Vector Lab, Inc., Burlingame, USA). Slides were again washed in PBS and color was developed by 5 min incubation in diaminobezidine (DAB) solution. Slides were counterstained with H\&E. For Ki67 staining, Novared kit (Vector Lab Inc., Burlingame, USA) was used to develop red color for $5 \mathrm{~min}$.

For whole tumor analysis, the slides were fixed in acetone for $20 \mathrm{~min}$, then blocked with the primary antibody dilution buffer for $30 \mathrm{~min}$. The slides were incubated with the primary antibody for $1 \mathrm{~h}$ : rat anti-mouse CD31 (BD, dilution 1:200). After washing with PBS, the slides were incubated with Cy2-conjugated donkey anti-rat (Jackson Immunoresearch, 1:100) for $30 \mathrm{~min}$, washed with PBS and fixed in 4\% PFA for $15 \mathrm{~min}$. The slides were immediately imaged.

Image collection and analysis

The tumor sections were quantitatively analyzed with a method based on a computerized digital image-analysis system. A high-resolution, intensified solid-state camera on a fluorescence microscope (Axioskop [Zeiss, Germany]) was used. The images were recorded, processed, and analyzed using the digital imaging application Axiovision. For quantitation purposes, Adobe Photoshop and MATLAB (MathWorks, Inc., Natick, MA) were used. Histologic analysis was not performed on areas of oxygen probe measurement to avoid areas that might have been damaged by probe placement.

$V E G F$. After the slides were scanned at low magnification $(40 \times)$, three areas (per animal) of maximum VEGF expression were selected $(400 \times)$. The degree of positive VEGF staining on the section was assessed at low magnification $(40 \times)$. The percentage of tumor cells staining for VEGF was analyzed by measuring positively stained pixels/total pixels. The mean value of the examined fields was calculated (and grouped together to represent each animal).

Ki67. After the slides were scanned at low magnification $(40 \times)$, the cells with positively stained nuclei were counted in three fields at a magnification of $400 \times$. Total number of tumor cells was counted in each field and Ki67 index was calculated as the percentage of positive cells/total cells.

Hypoxia evaluation. The slides were scanned at low magnification $(40 \times)$ using a TRITC filter to identify three hypoxic areas that were captured. The hypoxic fraction was calculated as the area of hypoxic staining divided by the total area. Necrotic areas within each section were excluded on the basis of H\&E staining.

Whole tumor analysis. The slides were imaged using a computer controlled scanning stage and later "stitched" together to gain one image of the entire tumor. Fluorescence images were acquired with a monochromatic CCD camera and $10 \times$ objective (pixel size $=1.48$ by $1.48 \mu \mathrm{m}$ ). Color images were acquired with a color CCD camera and a $5 \times$ objective (pixel size $=0.69$ by $0.69 \mu \mathrm{m}$ ). These images for each tumor were aligned in Adobe Photoshop and a series of masks were created: tumor mask, necrotic mask, and necrotic edge mask. These masks and the original fluorescence image of tumor blood vessels $\left(\mathrm{CD} 31^{+}\right)$, isolated with a thresholding procedure [20], were analyzed with custom Matlab software to calculate the necrotic fraction, MVD, median distance to the vascular surface (MDVS) from viable tumor and the median distance to the 
necrotic edge (MDNE) from the vascular surface. The MDVS describes the vascular spatial frequency and architecture, where a shorter distance indicates a better organized vascular network for oxygen delivery. MDNE represents a ratio of supply to demand convolved with the inherent cell disposition to die. These data are presented as the median distance \pm SEM.

The distance from each pixel to the nearest blood vessel was calculated in Matlab and displayed as a cumulative frequency of distances. This distance represents the minimum distance that a solute (e.g., oxygen) must travel from the blood vessel to a tumor cell such that larger values suggest that a solute has a larger distance to travel in order to reach that tumor cell. For a constant amount of tumor cell consumption and vascular concentration, larger distances indicated a vascular network that is not as efficient for transport as a vascular network with shorter distances.

For all immunohistochemical endpoints, the observers were blinded to the treatment groups during data acquisition.

\section{Statistics}

Statistical analyses were done similarly for all endpoints. Tumor growth and oxygenation data were compared using Student's $t$ test. Endpoints for each tumor line, MVD, pimonidazole, VEGF, and Ki67 immunohistochemistry were evaluated with a global ANOVA with treatment group as the factor and a Fisher's PLSD post hoc test (Statview, SAS Institute, Cary, NC). Values are expressed as mean \pm SEM. $P$ values of $<0.05$ were considered statistically significant. All statistical tests were two sided.

\section{Results}

Her2 blockade increases tumor oxygenation

Treatment with trastuzumab over 2 weeks, as predicted, statistically significantly reduced tumor size in $\mathrm{MCF}^{\mathrm{Her} 2}$ tumors (mean volume of control MCF7 ${ }^{\text {Her2 }}$ tumors was $235.3 \mathrm{~mm}^{3}$ vs $62.5 \mathrm{~mm}^{3}$ for trastuzumab-treated tumors; $P<0.05 ; t$ test). There was no statistically significant effect of Her2 blockade on MCF7 ${ }^{\text {WT }}$ tumor growth, but there was a trend toward smaller tumors in the trastuzumab-treated group (Table 1). This may be explained by the trend in proliferation index (below).

Tumor oxygenation status was determined by direct $\mathrm{pO}_{2}$ measurements. When measured directly, trastuzumabtreated animals with $\mathrm{MCF} 7^{\mathrm{Her} 2}$ tumors $(N=5)$ had a mean $\mathrm{pO}_{2}$ of $16 \mathrm{mmHg} \pm 0.79$, whereas control-treated animals with $\mathrm{MCF} 7^{\mathrm{Her} 2}$ tumors $(N=5)$ had a lower $\mathrm{pO}_{2}$ of $12 \mathrm{mmHg} \pm 0.75$ (Table 1 ). $\mathrm{MCF}^{\mathrm{Her} 2}$ tumors treated with trastuzumab also had a lower fraction of $\mathrm{pO}_{2}$ measurements less than $10 \%$ (41\% in the trastuzumab-treated group vs $62 \%$ in the control-treated group). The difference was statistically significant $(P<0.05 ; t$ test $)$ indicating that Her2 blockade in $\mathrm{MCF}^{\mathrm{Her} 2}$ tumors improved tumor oxygenation. Mean $\mathrm{pO}_{2}$ values were not significantly different between $\mathrm{MCF} 7{ }^{\mathrm{WT}}$ tumors treated with trastuzumab $(N=5)$ or control $(N=5), 21 \mathrm{mmHg} \pm 5.9$ and $23 \mathrm{mmHg} \pm 3.2$, respectively $\left(P>0.05 ; t\right.$ test). $\mathrm{MCF}^{\mathrm{Her} 2}$ tumors were statistically significantly more hypoxic $(12 \mathrm{mmHg})$ than the MCF7 ${ }^{\mathrm{WT}}$ tumors $(23 \mathrm{mmHg})$, suggesting that Her2 overexpression results in increased tumor hypoxia $(P<0.05 ; t$ test). Her2 overexpression also resulted in a higher fraction of $\mathrm{pO}_{2}$ measurements below $10 \%\left(62 \%\right.$ in the $\mathrm{MCF}^{\mathrm{Her} 2}$ control-treated tumors vs $26 \%$ in the MCF7 ${ }^{\mathrm{WT}}$ controltreated tumors, Table 1).

Determinations of hypoxia were also done using pimonidazole staining of tumor sections. Quantitation of immunohistochemical staining of tumor sections revealed patterns similar to the above direct oxygenation measurements. Control MCF7 ${ }^{\mathrm{WT}}$ tumors had a mean hypoxic fraction of 0.22 , while that of trastuzumab-treated $\mathrm{MCF}^{\mathrm{WT}}$ tumors was 0.29 ( $P=0.04$; ANOVA). In contrast, control MCF7 ${ }^{\text {Her2 }}$ tumors had a mean hypoxic fraction of 0.64 , while trastuzumab treatment significantly

Table 1 Tumor pO2

\begin{tabular}{lllll}
\hline & MCF7 $^{\mathrm{WT}}$ control & MCF7 $^{\mathrm{WT}}$ trastuzumab & MCF7 $^{\text {Her2 }}$ control & MCF7 $^{\text {Her2 }}$ trastuzumab \\
\hline Mean $\mathrm{pO}_{2}(\mathrm{mmHg})$ & $23 \pm 3.2$ & $21 \pm 5.9$ & $12 \pm 0.75^{*}$ & $16 \pm 0.79 * *$ \\
$\% \mathrm{pO}_{2}<10 \mathrm{mmHg}$ & $29 \pm 7.6$ & $26 \pm 3.9$ & $62 \pm 2.5^{* * *}$ & $41 \pm 4.6^{\dagger}$ \\
Mean endpoint tumor volume $\left(\mathrm{mm}^{3}\right)$ & $313 \pm 59$ & $249 \pm 33$ & $235 \pm 29$ & $62 \pm 15^{\dagger \dagger}$ \\
$n$ & 5 & 5 & 5 & 5 \\
\hline
\end{tabular}

* $\mathrm{MCF}^{\mathrm{WT}}$ vs $\mathrm{MCF}^{\mathrm{Her} 2}$ control-treated; $P<0.05 ; t$ test

** $\mathrm{MCF}^{\mathrm{Her} 2}$ control-treated vs $\mathrm{MCF} 7^{\mathrm{Her} 2}$ trastuzumab-treated; $P<0.05 ; t$ test

*** $\mathrm{MCF}^{\mathrm{Her} 2}$ control-treated vs $\mathrm{MCF} 7^{\mathrm{WT}}$ control-treated; $P<0.05 ; t$ test

$\dagger \mathrm{MCF}^{\mathrm{Her} 2}$ control-treated vs $\mathrm{MCF}^{\mathrm{Her} 2}$ trastuzumab-treated; $P<0.05 ; t$ test

$\dagger \dagger \mathrm{MCF} 7^{\mathrm{Her} 2}$ control-treated vs MCF $7^{\mathrm{Her} 2}$ trastuszumab-treated; $P<0.05 ; t$ test

$\mathrm{pO}_{2}$ measurements were done immediately after the 2 week treatment using an Oxylite fiber-optic oxygen probe. The mean pO2 values are given in $\mathrm{mmHg} \pm$ standard deviation 
reduced the hypoxic fraction to $0.46 \quad(P=0.016$; ANOVA). $\mathrm{MCF}^{\mathrm{Her} 2}$ control-treated tumors were statistically significantly more hypoxic than $\mathrm{MCF}^{\mathrm{WT}}$ controltreated tumors (Table 2, Fig. 2a, d; $P=0.001$; ANOVA).

Her2 blockade inhibits tumor cell proliferation

Her2 overexpression in this MCF7 tumor cell line increased proliferation index. Based on Ki67 staining, control-treated $\mathrm{MCF} 7^{\mathrm{WT}}$ cells had a mean proliferation index in vivo of $51 \pm 2 \%$, while $\mathrm{MCF} 7^{\mathrm{Her} 2}$ control-treated cells had a mean proliferation index of $70 \pm 3 \%$ (Table 2, Fig. $2 \mathrm{~d} ; P=0.001$; ANOVA).

Her2 blockade was effective in reducing the proliferation index. $\mathrm{MCF}^{\mathrm{Her} 2}$ tumors treated with trastuzumab had a mean proliferation index of $47 \pm 3 \%$, whereas in control tumors, the mean proliferation index was $60 \pm 3 \%$ (Table 2, Fig. 2b, d). This represents a significant decrease in proliferative cells in the trastuzumab-treated $\mathrm{MCF} 7^{\mathrm{Her} 2}$ tumors when compared with controls $(P=0.03$; ANOVA). Trastuzumab- and control-treated MCF ${ }^{\text {WT }}$ tumors did not have significantly different proliferative indices, $44 \pm 3$ and $51 \pm 2 \%$, respectively $(P=0.10$; ANOVA), although there is a clear trend that corresponds to a trend in smaller tumors in the trastuzumab-treated group.

\section{Her2 blockade improves tumor vascular architecture}

Mean percent area positive for VEGF was lower in $\mathrm{MCF} 7^{\mathrm{Her} 2}$ tumors treated with trastuzumab vs controls (35 \pm 11 and $76 \pm 1 \%$, respectively; Table 2, Fig. 2c). These values represent a statistically significant reduction in VEGF expression $(P=0.02 ; t$ test $)$. Mean VEGF staining was not different in trastuzumab-treated and control $\mathrm{MCF}^{\mathrm{WT}}$ tumors: $25 \pm 4$ and $25 \pm 2 \%$, respectively $(P=0.41 ; t$ test).

An evaluation of the vascular architecture was accomplished using whole tumor images (Fig. 3a, b). In addition, vascular density was determined from high magnification images $(400 \times)$ of CD31-stained tumor sections. Her2 signaling blockade in $\mathrm{MCF}^{\mathrm{Her} 2}$ tumors resulted in significantly increased vascular density from $84 \pm 12$ vessels per $\mathrm{mm}^{2}$ in control tumors to $150 \pm 28$ in trastuzumabtreated tumors (Table 3, Fig. 4a; $P=0.01$; Fisher's PLSD). Vascular density was not significantly different between control $\mathrm{MCF}^{\mathrm{WT}}$ and $\mathrm{MCF}^{\mathrm{Her} 2}$ tumors, as well as between control- and trastuzumab-treated $\mathrm{MCF} 7^{\mathrm{WT}}$ tumors $(P>0.05$, Fisher's PLSD).

The median distance to vascular surface (MDVS) was relatively similar for both trastuzumab-treated and control $\mathrm{MCF}^{\mathrm{WT}}$ tumors, as well as for both control $\mathrm{MCF} 7^{\mathrm{WT}}$ and $\mathrm{MCF}^{\mathrm{Her} 2}$ tumors (Table 3, Fig. 4b). However, Her2 blockade in $\mathrm{MCF}^{\mathrm{Her} 2}$ tumors reduced the MDVS to $35 \pm 4$ compared to a MDVS of $47 \pm 4 \mu \mathrm{m}$ in control $\mathrm{MCF}^{\mathrm{Her} 2}$ tumors, resulting in tumors with more evenly spaced blood vessels and shorter distances between blood vessels and tumor cells. This relationship is also represented in a cumulative frequency plot in Fig. 4c. This difference in vascular architecture indicates that trastuzumab treated $\mathrm{MCF}^{\mathrm{Her}}$ tumors had a more efficient vascular architecture for oxygen transport than control $\mathrm{MCF}^{\mathrm{Her} 2}$ tumors.

Her2 blockade in $\mathrm{MCF}^{\mathrm{Her}}$ tumors exhibited a trend in the median distance to necrotic edge (MDNE). The MDNE in control $\mathrm{MCF}^{\mathrm{Her}}$ tumors was $97 \pm 11$, however, in trastuzumab-treated $\mathrm{MCF}^{\mathrm{Her} 2}$ tumors this distance was reduced to $62 \pm 11 \mu \mathrm{m}$ (Table 3, Fig. 4b). MDNE was not statistically different between control $\mathrm{MCF}^{\mathrm{WT}}$ and $\mathrm{MCF} 7^{\mathrm{Her} 2}$ or trastuzumab-treated $\mathrm{MCF} 7^{\mathrm{WT}}$ tumors. Considering that

Table 2 Immunohistochemical analysis of tumors

\begin{tabular}{lcccc}
\hline & MCF7 $^{\text {WT }}$ control & MCF7 $^{\text {WT }}$ trastuzumab & MCF7 $^{\text {Her2 }}$ control $^{\text {MCF7 }}{ }^{\text {Her2 }}$ trastuzumab $^{\text {Mrum }}$ \\
\hline Hypoxic fraction (pimonidazole) & $0.22 \pm 0.04$ & $0.29 \pm 0.06$ & $0.64 \pm 0.08^{*}$ & $0.46 \pm 0.04^{* *}$ \\
VEGF expression \% area (+) & $25 \pm 2$ & $25 \pm 4$ & $76 \pm 1^{* * *}$ & $35 \pm 11^{\dagger}$ \\
Proliferation index (Ki67) & $51 \pm 2$ & $44 \pm 3$ & $60 \pm 3^{\dagger \dagger}$ & $47 \pm 3^{\dagger \dagger}$ \\
\hline
\end{tabular}

Hypoxic fraction is reported as the percentage of pimonidazole-stained tumor cells over the total number of cells in the tumor section $\times 100 \pm$ SEM. Relative VEGF expression is reported as the ratio of VEGF stained area over the total tumor parenchymal area $\times 100 \pm$ SEM. Proliferation index is reported as the number of positively staining nuclei over the total number of nuclei in the field $\times 100 \pm$ SEM

$* \mathrm{MCF}^{\mathrm{WT}}$ vs MCF7 ${ }^{\mathrm{Her} 2}$ control-treated; $P=0.001 ;$ ANOVA

** $\mathrm{MCF}^{\mathrm{Her} 2}$ control-treated vs $\mathrm{MCF}^{\mathrm{Her} 2}$ trastuzumab-treated; $P=0.016$; ANOVA

*** $\mathrm{MCF}^{\mathrm{WT}}$ vs MCF7 ${ }^{\mathrm{Her} 2}$ control-treated; $P=0.0001$; ANOVA

$\uparrow \mathrm{MCF}^{\mathrm{Her} 2}$ control-treated vs $\mathrm{MCF}^{\mathrm{Her} 2}$ trastuzumab-treated; $P=0.02$; ANOVA

$\dagger \dagger \mathrm{MCF}^{\mathrm{WT}}$ control-treated vs $\mathrm{MCF}^{\mathrm{Her} 2}$ control-treated; $P=0.001$; ANOVA

$\dagger \dagger \mathrm{MCF}^{\mathrm{Her} 2}$ control-treated vs $\mathrm{MCF}^{\mathrm{Her} 2}$ trastuzumab-treated; $P=0.03$; ANOVA 
Fig. 2 Tumor panel immunohistochemistry and quantitation. Her2 blockade improves tumor oxygenation, inhibits tumor cell proliferation, and decreases VEGF expression. Shown are highmagnification $(400 \times)$ representative images from immunohistochemical staining of $\mathrm{MCF}^{\mathrm{WT}}$ (control- and trastuzumab-treated) and $\mathrm{MCF}^{\mathrm{Her} 2}$ (control- and trastuzumab-treated) tumor sections. Assessments for tumor: a hypoxia

(pimonidazole), b proliferation (Ki67), and c VEGF levels were completed. Scale

bars $=100 \mu \mathrm{m}$. Quantitation of staining is shown in d. Hypoxia is reported as the percentage of tumor cells staining positive for pimonidazole. Proliferation index is reported as the number of Ki67 stained nuclei over the total number of nuclei $\times 100$. VEGF relative expression is reported as the ratio of VEGF stained area over the total tumor parenchymal area $\times 100$. Error bars represent SEM

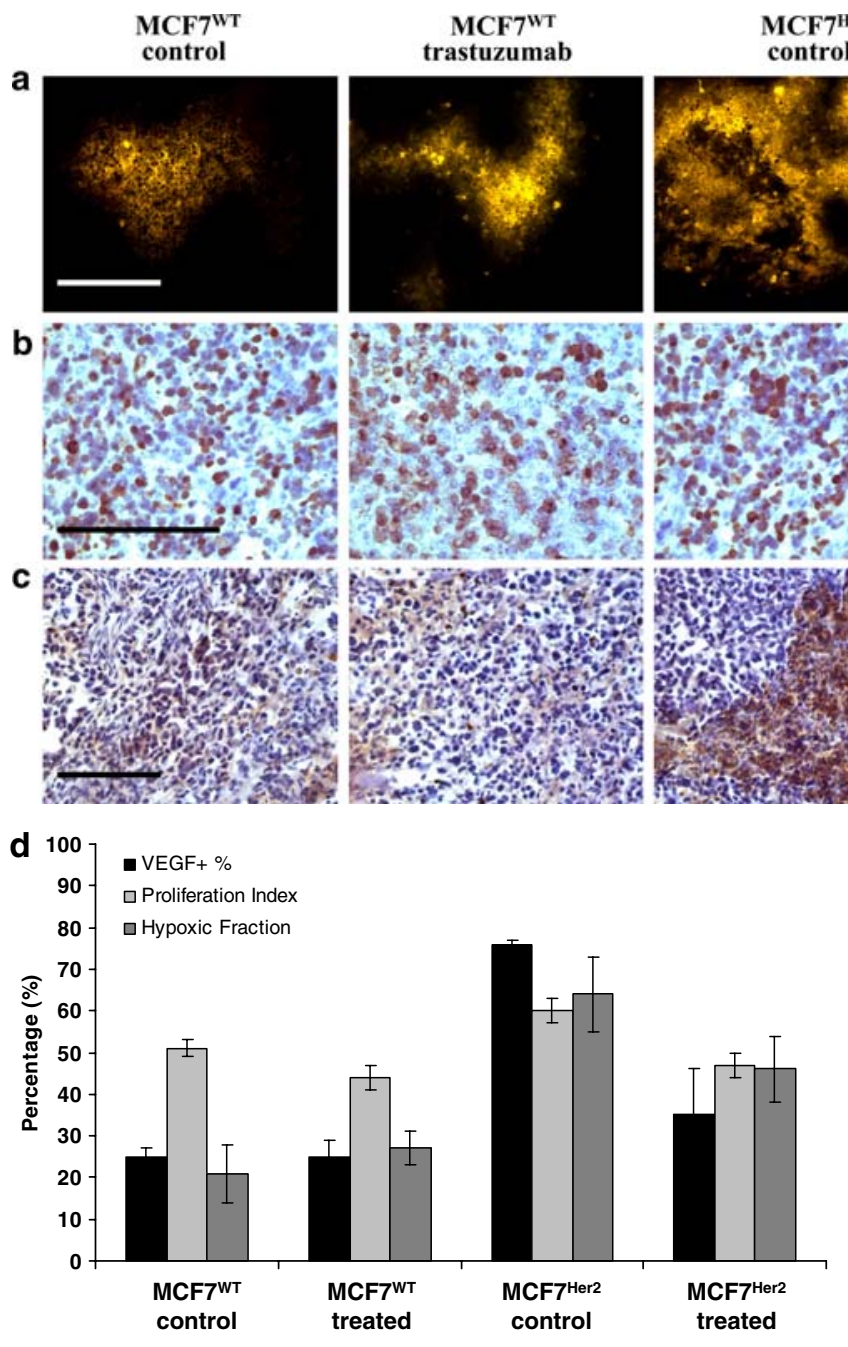

trastuzumab treated $\mathrm{MCF}^{\mathrm{Her} 2}$ tumors had a greater MVD than other tumors, this shorter distance to the necrotic edge suggests that the trastuzumab treated $\mathrm{MCF} 7^{\mathrm{Her} 2}$ tumors were more susceptible to death from nutrient depletion, as compared with the other experimental groups.

\section{Discussion}

Oxygen consumption rate, vascular density and vascular architecture are among the main physiologic determinants of oxygen transport in tumors $[10,21]$. In this paper, we demonstrate that all three features are affected by Her $2 /$ neu overexpression. Several clinical reports indicate that increased tumor cell proliferation is associated with overexpression of Her2 [13-16]. Her2 overexpression can also result in increased blood vessel growth as a result of its influence on VEGF expression [18, 19]. The balance between these two functions of Her2 will likely dictate the oxygenation status of Her2 positive tumors under baseline conditions and in response to Her2 function blockade.
The results of this study indicate that Her2 overexpression creates a more hypoxic environment, reflected by both oxygen probe and pimonidazole immunohistochemistry measurements. The poorer oxygenation is a result of either inefficiencies in oxygen transport created by lower vascular density, more irregular vascular geometry or tumor cell proliferation [10]. The results of the MDVS are helpful in this regard. This value was not different between wild type and Her2 overexpressing tumors. This indicates that the vascular architecture, as assessed by this method, cannot explain the higher hypoxic fraction in the Her2 overexpressing tumors. Therefore, one can conclude that the main determinant of the greater degree of hypoxia is the higher growth fraction, i.e., tumor cell proliferation.

Simulations of oxygen transport in tumor models, based on experimental measurements of vascular architecture and other relevant parameters have indicated that large changes in oxygenation can be induced by as little as $30 \%$ change in oxygen consumption rate [9]. This change in oxygen consumption rate is in the order of magnitude seen in this study. The proliferation rate for wild type MCF7 cells in 


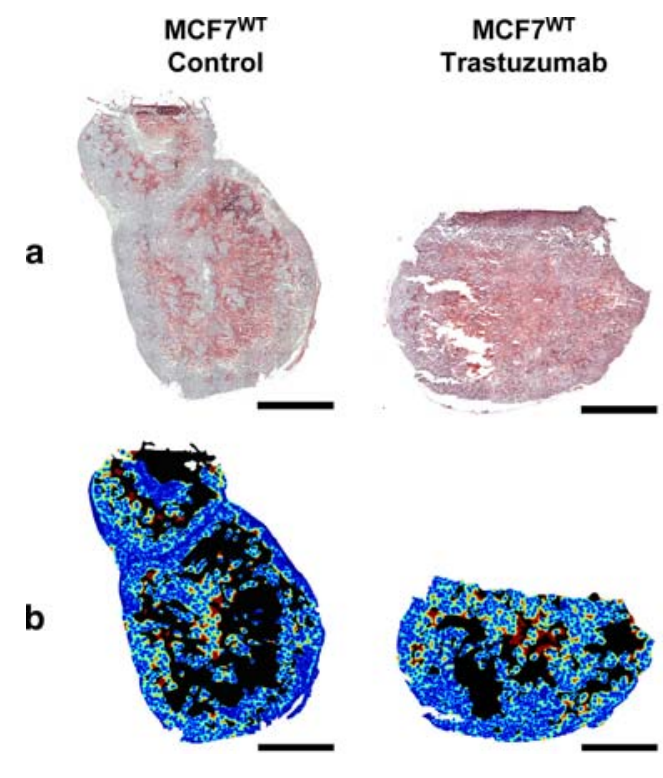

Fig. 3 Whole tumor vessel analysis. Shown are whole tumor representative images from immunohistochemical staining of $\mathrm{MCF}^{\mathrm{WT}}$ (control- and trastuzumab-treated) and $\mathrm{MCF} 7^{\mathrm{Her} 2}$ (controland trastuzumab-treated) tumors. In a, whole tumor images of $\mathrm{H} \& \mathrm{E}$ staining are shown. The distance map is shown in $\mathbf{b}$ where a distance of zero is dark blue (online) and a distance of $200 \mu \mathrm{m}$ is shown as

vivo was around $50 \%$ and this increased to approximately $70 \%$ in Her2 overexpressing tumors. Oxygen consumption rates for proliferating cells are approximately 3-5-fold higher than that of quiescent cells $[12,22]$. This $20 \%$ difference in proliferation could easily induce a large difference in hypoxia based on previous modeling by Secomb et al. [10].

To illustrate this point, we describe a relatively straightforward way to estimate differences in oxygen consumption rate, based on differences in the proliferative fraction. Relative metabolic rates (compared with a tumor with a proliferative fraction of zero) were estimated for each experimental group using the following equation:
MCF7Her2

Control
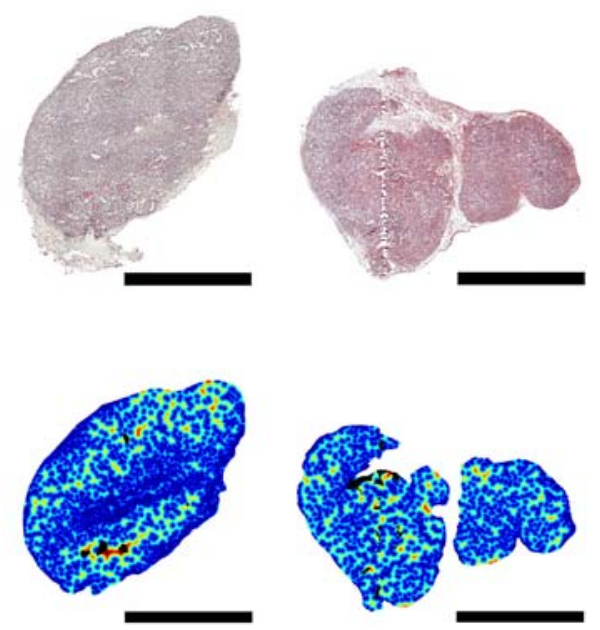

dark red (online) (all distances $>200 \mu \mathrm{m}$ are shown as dark red). In $\mathrm{MCF} 7$ Her2 tumors, treatment with trastuzumab results in a "normalization" of the vascular architecture, illustrated by a more regular distribution of vascular distances in $\mathbf{b}$. The necrotic area is indicated by black regions and non-tumor area is white. The scale bar is $3 \mathrm{~mm}$

Relative Metabolic Rate $=($ proliferative fraction $) \times 4$ + (non-proliferative fraction) $\times 1$

The multipliers of 4 and 1 come from the average difference in oxygen consumption rates for proliferative and quiescent cells, as referenced above (Table 4). Using these numbers, it is possible to compare oxygen consumption rates between experimental groups. The ratio of relative oxygen consumption rates between control $\mathrm{MCF}^{\mathrm{WT}}$ and $\mathrm{MCF}^{\mathrm{Her} 2}$ tumors is $2.80 / 2.53=1.11$, indicating that the oxygen consumption rate of the Her 2 overexpressing line is about $10 \%$ higher than the wild type line. In the analysis by

Table 3 Summary of vessel analysis

\begin{tabular}{|c|c|c|c|c|}
\hline & $\mathrm{MCF}^{\mathrm{WT}}$ control & $\mathrm{MCF}^{\mathrm{WT}}$ trastuzumab & $\mathrm{MCF}^{\mathrm{Her} 2}$ control & $\mathrm{MCF}^{\mathrm{Her} 2}$ trastuzumab \\
\hline Total tumor area $\left(\mathrm{mm}^{2}\right)$ & $35.4 \pm 12.2$ & $34.1 \pm 8.8$ & $21.4 \pm 11.6^{\mathrm{NS}}$ & $6.5 \pm 3.1^{\mathrm{NS}}$ \\
\hline Necrotic fraction & $0.24 \pm 0.08$ & $0.18 \pm 0.09$ & $0.06 \pm 0.03^{\mathrm{NS}}$ & $0.07 \pm 0.05^{\mathrm{NS}}$ \\
\hline Vascular density (vessels $/ \mathrm{mm}^{2}$ ) & $95 \pm 8$ & $82 \pm 7$ & $84 \pm 12^{\mathrm{NS}}$ & $150 \pm 28^{*}$ \\
\hline Area per vessel $\left(\mu \mathrm{m}^{2}\right)$ & $258 \pm 31$ & $206 \pm 30$ & $250 \pm 32$ & $246 \pm 19$ \\
\hline Median distance to vascular surface (MDVS; mm) & $47.4 \pm 4$ & $50.5 \pm 3$ & $47 \pm 4$ & $35 \pm 4^{\mathrm{NS}}$ \\
\hline Median distance to necrotic edge (MDNE; mm) & $95 \pm 11$ & $97 \pm 5$ & $97 \pm 11$ & $62 \pm 11^{\mathrm{NS}}$ \\
\hline
\end{tabular}

A variety of vessel analyses were performed on $\mathrm{MCF} 7^{\mathrm{WT}}$ and $\mathrm{MCF}^{\mathrm{Her} 2}$ tumors treated with control and trastuzumab, including total tumor area $\left(\mathrm{mm}^{2}\right)$, necrotic fraction, vascular density $\left(\right.$ vessels $\left./ \mathrm{mm}^{2}\right)$, area per vessel $\left(\mu \mathrm{m}^{2}\right)$, median distance to vascular surface (MDVS; $\left.\mu \mathrm{m}\right)$ and median distance to necrotic edge (MDNE; $\mu \mathrm{m})$

* MCF7Her2 trastuzumab-treated vs MCF7Her2 control-treated; $P=0.03$; ANOVA

NS Not statistically significant; $P>0.05$ 

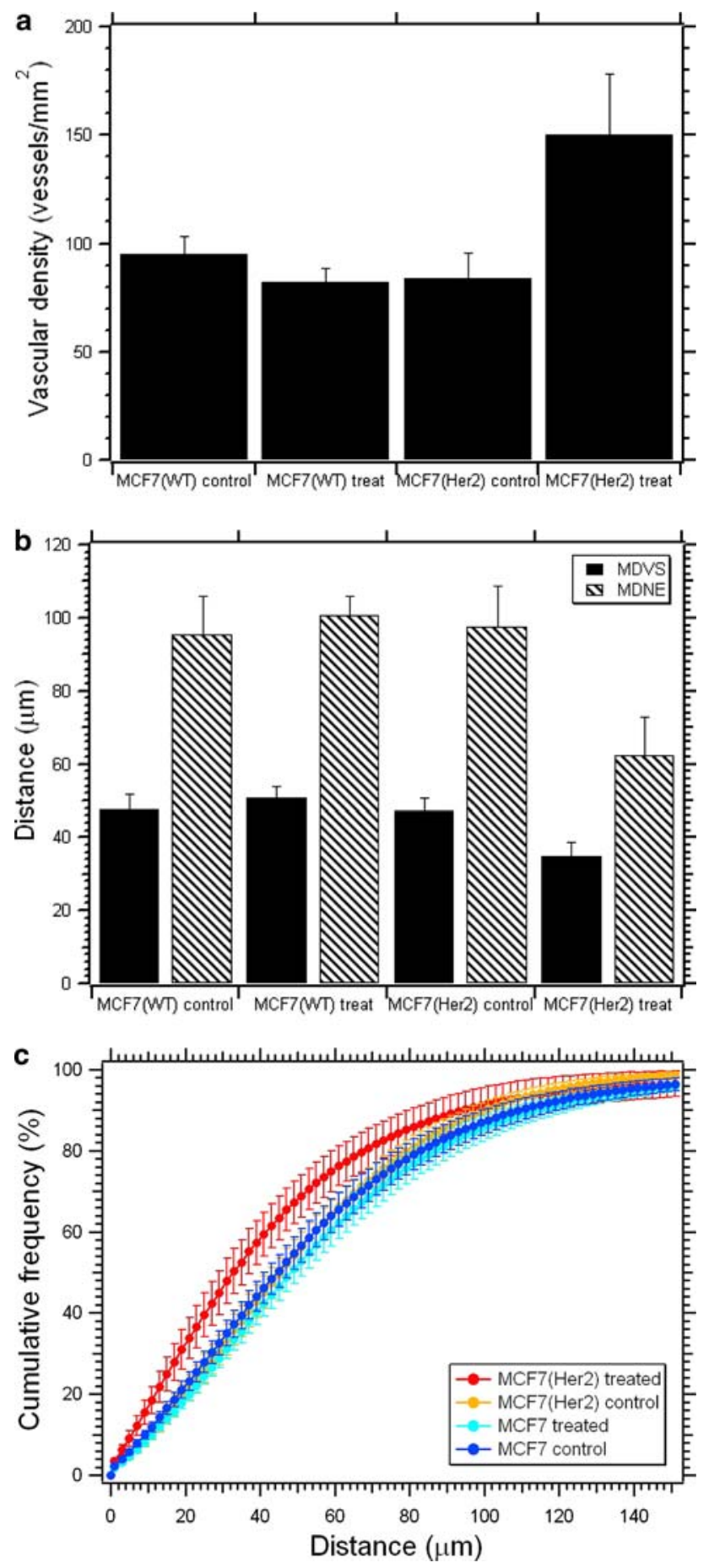

Fig. 4 Tumor vascular spatial frequency and architecture. Her2 overexpression creates sparser and more unevenly spaced vascular networks. Treatment of $\mathrm{MCF} 7^{\mathrm{Her} 2}$ tumors with trastuzumab restored the tumor vasculature spatial frequency and distribution to $\mathrm{MCF} 7^{\mathrm{WT}}$ tumor's values, demonstrated by increased vascular density (a), decreased median distance to vascular surface (MDVS) and median distance to necrotic edge (MDNE) (b), and a shift in cumulative frequency to higher distance values $(\mathbf{c})$
Table 4 Relative metabolic rates

\begin{tabular}{ll}
\hline Treatment group & Relative metabolic rate \\
\hline $\mathrm{MCF}^{\mathrm{WT}}$ control & 2.53 \\
$\mathrm{MCF7}^{\mathrm{WT}}$ trastuzumab & 2.32 \\
$\mathrm{MCF}^{\mathrm{Her} 2}$ control & 2.80 \\
$\mathrm{MCF}^{\mathrm{Her} 2}$ trastuzumab & 2.41
\end{tabular}

See "Discussion" for details on how these values were calculated

Secomb, a $30 \%$ reduction in oxygen consumption rate was successful in reducing the hypoxic fraction from $12 \%$ to zero. In the current analysis, there is a $40 \%$ difference in hypoxic fraction between the two control groups. Because the difference in hypoxic fraction is so much greater than the $12 \%$ reduction predicted by Secomb for a $30 \%$ drop in oxygen consumption rate, we suggest that factors other than oxygen consumption rate are playing a role. It is possible that the Her2 overexpressing line is more metabolically active because of other factors aside from proliferation-such as differences in cell motility and invasion. Alternatively there may be other differences in the ability of the microvasculature to deliver oxygen efficiently. We are not able to discern from our data, for example, whether there are differences in shunt flow between these tumor types, which could very well be the case in the Her2 overexpressing line, since it has a significantly higher level of VEGF expression, which would tend to lead to higher diameter blood vessels [23].

When trastuzumab was administered to Her2 overexpressing tumors, there were significant reductions in cell proliferation, VEGF expression, an increase in vascular density and a trend toward an improvement in vascular architecture, reflected by the shortening of MDVS. This result is consistent with a duality of effects induced by trastuzumab treatment in the MCF7 Her2 overexpressing line. The reduction in proliferation likely caused a reduction in oxygen consumption rate and the increase in vascular density likely improved oxygen delivery.

The ratio of relative oxygen consumption rates for $\mathrm{MCF}^{\mathrm{WT}}$ tumors was 1.09 (control-treated/trastuzumabtreated). The proliferation index was not statistically significantly different between $\mathrm{MCF} 7^{\mathrm{WT}}$ groups, which is consistent with there being no significant difference in the degree of hypoxia between these two groups., In contrast, the proliferation rate in the $\mathrm{MCF}^{\mathrm{Her} 2}$ tumors after treatment with trastuzumab was significantly lower than the control, yielding a ratio of relative oxygen consumption rates of 1.16. This difference in oxygen consumption correlated with a $20 \%$ drop in hypoxic fraction, measured by both pimonidazole immunohistochemistry and the probe 
measurement method. We cannot rule out that the increase in microvessel density induced by trastuzumab treatment may also play a role in influencing the degree of hypoxia, however.

Additional work is required to completely resolve which of these additional mechanisms might be in play to explain the differences in hypoxic fraction. We believe that simple measurement of oxygen consumption rate would be insufficient to explain the differences, however, because factors such as differences in motility and invasion could not be taken into account using standard methods, which require measurement of cells in suspension culture in sealed tubes [24].

Our findings appear to be in contrast to studies showing no effects of trastuzumab on the fraction of viable hypoxic Her2 + tumor cells [25]. In this study, Warburton et al. examined the effects of trastuzumab alone and in combination with gefitinib, a selective small molecule inhibitor of EGFR. However, one main difference in our study lies in our measurements of tumor oxygenation in vivo, as well as by immunohistochemistry, whereas previous studies determined hypoxia in single-cell suspensions by flow cytometry.

An unexpected additional observation was a trend in the reduction in distance to nearest necrotic edge. These results suggest that trastuzmab treated Her2 overexpressing cells may be more sensitive to nutrient withdrawal than the untreated control and wildtype cells, given that their oxygenation was improved. This may reflect a lowered capacity to switch from aerobic to anaerobic metabolism, resulting in lower threshold for cell death [26]. We have shown previously that both oxygen and glucose gradients contribute to nutrient depletion and necrosis [27]. Additional studies to examine nutrient gradients in these tumor models might yield mechanistic information underlying the contribution of nutrient depletion to necrosis.

There have been conflicting reports on the effects of trastuzumab on tumor vasculature. Dragowska et al. demonstrated increased VEGF levels and an increased viable hypoxic cell fraction in tumors subjected to Her2 blockade, but failed to demonstrate a difference in tumor vascularization [28]. Izumi et al. examined the effects of Her2 blockade on blood vessel diameter, length, volume and permeability in Her2(+) mouse breast tumors [29]. They demonstrated a reduction in diameter, vascular volume, and permeability (but not length) of tumor blood vessels, which they coined the "normalization" of tumor blood vessels [30]. Our result is somewhat consistent with this prior work. In this model system, the pruning of vasculature following administration of trastuzumab to Her2 + tumors occurs at a pace that is slower than loss of tumor cells. This is reflected by a net increase in vascular density and decrease in MDVS. We clearly observed a reduction in overall VEGF levels, indicating that the increase in vascular density is not likely due to stimulation of angiogenesis. We show here that the improvement in oxygenation is a more complex function, involving a combination of cell loss from necrosis, reduced oxygen consumption and improved vascular architecture.

Trastuzumab is the first of a cohort of drugs that interfere with Her2 function. The small molecule tyrosine kinase inhibitor, lapatinib, combined with capecitabine was recently shown to prolong progression free survival in patients with Her2 positive locally advanced or metastatic breast cancer, who had already failed several other chemotherapy regimens [31]. These drugs will have wide ranging clinical applications for tumors that overexpress this receptor. It is important to keep in mind the changes in microenvironment created by Her2 blockade. The improvements in transport definitely increase oxygenation, but will also improve drug delivery as well. One would expect improved cell killing by radiation and a variety of traditional chemotherapeutic drugs that are known to be influenced by inefficiencies in delivery and hypoxia [32].

Open Access This article is distributed under the terms of the Creative Commons Attribution Noncommercial License which permits any noncommercial use, distribution, and reproduction in any medium, provided the original author(s) and source are credited.

\section{References}

1. Graeber TG, Osmanian C, Jacks T, Housman DE, Koch CJ, Lowe SW, Giaccia AJ (1996) Hypoxia-mediated selection of cells with diminished apoptotic potential in solid tumours. Nature 379:8891

2. Vaupel P, Harrison L (2004) Tumor hypoxia: causative factors, compensatory mechanisms, and cellular response. Oncologist 9(Suppl 5):4-9

3. Giaccia AJ (1996) Hypoxic stress proteins: survival of the fittest. Semin Radiat Oncol 6:46-58

4. Knowles HJ, Harris AL (2001) Hypoxia and oxidative stress in breast cancer. Hypoxia and tumourigenesis. Breast Cancer Res 3:318-322

5. Semenza GL (2000) Surviving ischemia: adaptive responses mediated by hypoxia-inducible factor 1 . J Clin Invest 106:809812

6. D'Angio CT, Finkelstein JN (2000) Oxygen regulation of gene expression: a study in opposites. Mol Genet Metab 71:371-380

7. Maxwell PH, Pugh CW, Ratcliffe PJ (2001) Activation of the HIF pathway in cancer. Curr Opin Genet Dev 11:293-299

8. Brown JM, Giaccia AJ (1998) The unique physiology of solid tumors: opportunities (and problems) for cancer therapy. Cancer Res 58:1408-1416

9. Secomb TW, Hsu R, Ong ET, Gross JF, Dewhirst MW (1995) Analysis of the effects of oxygen supply and demand on hypoxic fraction in tumors. Acta Oncol 34:313-316

10. Secomb TW, Hsu R, Braun RD, Ross JR, Gross JF, Dewhirst MW (1998) Theoretical simulation of oxygen transport to tumors by three-dimensional networks of microvessels. Adv Exp Med Biol 454:629-634 
11. Freyer JP, Sutherland RM (1985) A reduction in the in situ rates of oxygen and glucose consumption of cells in EMT6/Ro spheroids during growth. J Cell Physiol 124:516-524

12. Freyer JP (1994) Rates of oxygen consumption for proliferating and quiescent cells isolated from multicellular tumor spheroids. Adv Exp Med Biol 345:335-342

13. Sirvent JJ, Santafe M, Salvado MT, Alvaro T, Raventos A, Palacios J (1994) Hormonal receptors, cell proliferation fraction (Ki-67) and c-erbB-2 amplification in breast cancer. Relationship between differentiation degree and axillary lymph node metastases. Histol Histopathol 9:563-570

14. Shrestha P, Yamada K, Wada T, Maeda S, Watatani M, Yasutomi M, Takagi H, Mori M (1992) Proliferating cell nuclear antigen in breast lesions: correlation of c-erbB-2 oncoprotein and EGF receptor and its clinicopathological significance in breast cancer. Virchows Arch A Pathol Anat Histopathol 421:193-202

15. Horita K, Yamaguchi A, Hirose K, Ishida M, Noriki S, Imamura Y, Fukuda M (2001) Prognostic factors affecting disease-free survival rate following surgical resection of primary breast cancer. Eur J Histochem 45:73-84

16. Jeziorski A, Blonski JZ, Niewiadomska H (2000) The expression of products of oncogens c-erbB2 and EGFR and proliferating antigens Ki67 and PCNA in primary invasive ductal cancer of female breast. J Exp Clin Cancer Res 19:61-67

17. Petit AM, Rak J, Hung MC, Rockwell P, Goldstein N, Fendly B, Kerbel RS (1997) Neutralizing antibodies against epidermal growth factor and ErbB-2/neu receptor tyrosine kinases downregulate vascular endothelial growth factor production by tumor cells in vitro and in vivo: angiogenic implications for signal transduction therapy of solid tumors. Am J Pathol 151:1523-1530

18. Laughner E, Taghavi P, Chiles K, Mahon PC, Semenza GL (2001) HER2 (neu) signaling increases the rate of hypoxiainducible factor 1alpha (HIF-1alpha) synthesis: novel mechanism for HIF-1-mediated vascular endothelial growth factor expression. Mol Cell Biol 21:3995-4004

19. Li YM, Zhou BP, Deng J, Pan Y, Hay N, Hung MC (2005) A hypoxia-independent hypoxia-inducible factor-1 activation pathway induced by phosphatidylinositol-3 kinase/Akt in HER2 overexpressing cells. Cancer Res 65:3257-3263

20. Ostu N (1979) A threshold selection method from gray-level histograms. IEEE Trans Syst Man Cybernet 9:62-66

21. Secomb TW, Hsu R, Park EY, Dewhirst MW (2004) Green's function methods for analysis of oxygen delivery to tissue by microvascular networks. Ann Biomed Eng 32:1519-1529

22. Freyer JP, Tustanoff E, Franko AJ, Sutherland RM (1984) In situ oxygen consumption rates of cells in V-79 multicellular spheroids during growth. J Cell Physiol 118:53-61
23. Yuan F, Chen Y, Dellian M, Safabakhsh N, Ferrara N, Jain RK (1996) Time-dependent vascular regression and permeability changes in established human tumor xenografts induced by an anti-vascular endothelial growth factor/vascular permeability factor antibody. Proc Natl Acad Sci USA 93:1476514770

24. Snyder SA, Lanzen JL, Braun RD, Rosner G, Secomb TW, Biaglow J, Brizel DM, Dewhirst MW (2001) Simultaneous administration of glucose and hyperoxic gas achieves greater improvement in tumor oxygenation than hyperoxic gas alone. Int J Radiat Oncol Biol Phys 51:494-506

25. Warburton C, Dragowska WH, Gelmon K, Chia S, Yan H, Masin D, Denyssevych T, Wallis AE, Bally MB (2004) Treatment of HER-2/neu overexpressing breast cancer xenograft models with trastuzumab (Herceptin) and gefitinib (ZD1839): drug combination effects on tumor growth, HER-2/neu and epidermal growth factor receptor expression, and viable hypoxic cell fraction. Clin Cancer Res 10:2512-2524

26. Moeller BJ, Dreher MR, Rabbani ZN, Schroeder T, Cao Y, Li CY, Dewhirst MW (2005) Pleiotropic effects of HIF-1 blockade on tumor radiosensitivity. Cancer Cell 8:99-110

27. Schroeder T, Yuan H, Viglianti BL, Peltz C, Asopa S, Vujaskovic Z, Dewhirst MW (2005) Spatial heterogeneity and oxygen dependence of glucose consumption in R3230Ac and fibrosarcomas of the Fischer 344 rat. Cancer Res 65:5163-5171

28. Dragowska WH, Warburton C, Yapp DT, Minchinton AI, Hu Y, Waterhouse DN, Gelmon K, Skov K, Woo J, Masin D, Huxham LA, Kyle AH, Bally MB (2004) HER-2/neu overexpression increases the viable hypoxic cell population within solid tumors without causing changes in tumor vascularization. Mol Cancer Res 2:606-619

29. Izumi Y, Xu L, di Tomaso E, Fukumura D, Jain RK (2002) Tumour biology: herceptin acts as an anti-angiogenic cocktail. Nature 416:279-280

30. Jain RK (2001) Normalizing tumor vasculature with anti-angiogenic therapy: a new paradigm for combination therapy. Nat Med 7:987-989

31. Geyer CE, Forster J, Lindquist D, Chan S, Romieu CG, Pienkowski T, Jagiello-Gruszfeld A, Crown J, Chan A, Kaufman B, Skarlos D, Campone M, Davidson N, Berger M, Oliva C, Rubin SD, Stein S, Cameron D (2006) Lapatinib plus capecitabine for HER2-positive advanced breast cancer. N Engl J Med 355:27332743

32. Brown JM, Wilson WR (2004) Exploiting tumour hypoxia in cancer treatment. Nat Rev Cancer 4:437-447 